Rev. High Pressure Sci. Technol., Vol. 7 (1998) 787 789

\title{
Structures of Molecular Nitrogen at High Pressures
}

\author{
M. Hanfland, M. Lorenzen, C. Wassilew-Reul, and F. Zontone \\ European Synchrotron Radiation Facility, 38043 Grenoble, France
}

\begin{abstract}
We have studied structural properties of the high pressure phases of molecular nitrogen at room temperature using synchrotron radiation and angle dispersive powder diffraction with image plates as detectors. The cubic $\delta$-phase was found to be stable between 5 and $11 \mathrm{GPa}$. At $11 \mathrm{GPa}$ a phase transition to a new tetragonal phase was observed. Structural refinements suggest, that the transition is due to an orientational ordering of the disklike disordered molecules of the $\delta$-phase. At $16.5 \mathrm{GPa}$ the transition to the rhombohedral $\varepsilon$-phase occurred.

(molecular nitrogen, high pressure, synchrotron radiation, $\mathrm{x}$-ray diffraction, phase transitions)
\end{abstract}

\section{Introduction}

Like other diatomic molecules $\left(\mathrm{O}_{2}, \mathrm{CO}, \ldots\right)$ molecular nitrogen undergoes numerous structural phase transitions while varying temperature and pressure. At least five different crystalline modifications have been identified at pressures below $20 \mathrm{GPa}$. Nitrogen transforms from cubic $\alpha-\mathrm{N}_{2}$ (space group: $\mathrm{Pa} 3)$ [1] to tetragonal $\gamma-\mathrm{N}_{2} \quad\left(\mathrm{P}_{2} / \mathrm{mnm}\right)$ [1] to rhombohedral $\varepsilon-\mathrm{N}_{2}(\mathrm{R}-3 \mathrm{c})$ [2] with increasing pressure at low temperatures. All these phases are characterised by orientationally ordered nitrogen molecules. At high temperature two phases with orientational disordered molecules are known, hexagonal $\beta-\mathrm{N}_{2}\left(\mathrm{P}_{3} / \mathrm{mmc}\right)$ [1] and cubic $\delta-\mathrm{N}_{2}(\mathrm{Pm} 3 \mathrm{n})$ [3]. The cubic unit cell of the $\delta$-phase contains eight $\mathrm{N}_{2}$ molecules on two different crystallographic positions with a peculiar orientational disorder. While molecules centred at the 2(a) positions (corners and body centre of the unit cell) are spherically disordered like the molecules in the $\beta$-phase, molecules centred at the $6(d)$ positions are randomly orientated in a planar disk normal to the face of the unit cell (see e.g. Fig. 2 in [4]). At room temperature the $\beta-\delta$ phase transition takes place at $4.9 \mathrm{GPa}$. At $17 \mathrm{GPa}$ a transformation to another phase is observed, which is orientational ordered and believed to have the same structure as $\varepsilon-\mathrm{N}_{2}$. This is however in disagreement with Monte Carlo (MC) [4] and molecular dynamics (MD) [5] calculations, which favour tetragonal structures for the ordered phase. There is evidence for further structural phase changes from Raman spectroscopy $[6,7]$.

\section{Experiment}

Here we present an investigation of the structural properties of the high pressure phases of molecular nitrogen at room temperature using angle dispersive powder diffraction (ADX) with image plates as detectors. The measurements were performed in a diamond anvil cell at the ID9 beamline of the ESRF and cover the pressure range from 5 to $50 \mathrm{GPa}$. Samples were loaded by condensing nitrogen gas into the gasket hole of the diamond anvil cell at liquid nitrogen temperature. Pressures were measured by the ruby fluorescence method using the non-linear hydrostatic pressure scale [8]. X-rays from the U46 undulator of the beamline were focused vertically with a Pt-coated Si-mirror and horizontally with an asymmetrically cut bend $\mathrm{Si}(111)$ Laue monochromator to a beamsize of $30 \times 30 \mu \mathrm{m}^{2}$. Diffraction images were collected at wavelengths of $\lambda \approx 0.46 \AA(\omega \approx 27$ $\mathrm{keV})$. Samples were rocked by $\pm 3^{\circ}$ in $\omega$ and in some cases to further reduce texture in the diffraction images by $\pm 15^{\circ}$ in $\chi$. Exposure times were 5 to $15 \mathrm{~min}$. The image plates (size: A3) were scanned with $100 \mu \mathrm{m}$ resolution using a Molecular Dynamics STORM image plate reader. Sample to plate distances were 360 and $460 \mathrm{~mm}$ resulting in angular resolutions of $0.05^{\circ}$ and $0.04^{\circ}$, respectively. One dimensional diffraction pattern were obtained by integrating the two dimensional images with fit2d [9]. Structural parameters were refined using GSAS [10].

\section{Results}

Above $5 \mathrm{GPa}$ and below $11 \mathrm{GPa}$ the integrated diffraction pattern agreed with pattern expected for the cubic $\delta$-phase. A pattern measured at $9.5 \mathrm{GPa}$ is shown in Fig. 1. Besides the strong (200), (210) and (211) reflections all other reflections allowed within the cubic space group $\mathrm{Pm} 3 \mathrm{n}$ were observed to a maximum diffraction angle of $2 \theta=23^{\circ}$ (limited by the opening angle of the diamond anvil cell). The quality of the pattern was good enough to refine structural parameters of the $\delta$-phase. Taking into account preferred orientation an excellent agreement between the measured and the calculated pattern was achieved (Fig. 1). Structural parameters close to values found by Cromer et al. [3] in a single crystal diffraction study at $4.9 \mathrm{GPa}$ were obtained.

Above $11 \mathrm{GPa}$ additional diffraction lines appeared, the two strongest just above the (211) reflection of the $\delta$-phase, weaker ones between the (200) and (210) reflections and at higher diffraction angles. Non of these additional lines can be assigned to reflections allowed within the cubic space group $\mathrm{Pm} 3 \mathrm{n}$. A structural phase transition has taken place. A diffraction pattern of the new phase measured at $12.3 \mathrm{GPa}$ is shown in Fig. 2. The pattern can be indexed (Tab. 1) assuming a tetragonal unit cell containing $16 \mathrm{~N}_{2}$ molecules with lattice parameters related to the lattice parameter of the $\delta$-phase by $a_{\text {tet }} \approx a_{\text {cub }} \sqrt{2}$ and $c_{\text {tet }} \approx a_{\text {cub }}$. The unit cell is not identical to the unit cell of $\gamma-\mathrm{N}_{2}$. At the phase transition the tetragonal distortion is negligible. The c/a-ratio is $1 / \sqrt{2} \approx$ 0.707 within the accuracy of the measurement. With increasing pressure the distortion increases linearly the c/aratio reaching 0.702 at $16 \mathrm{GPa}$. The volume changes continuously during the transition. No anomalous behavior was observed. 


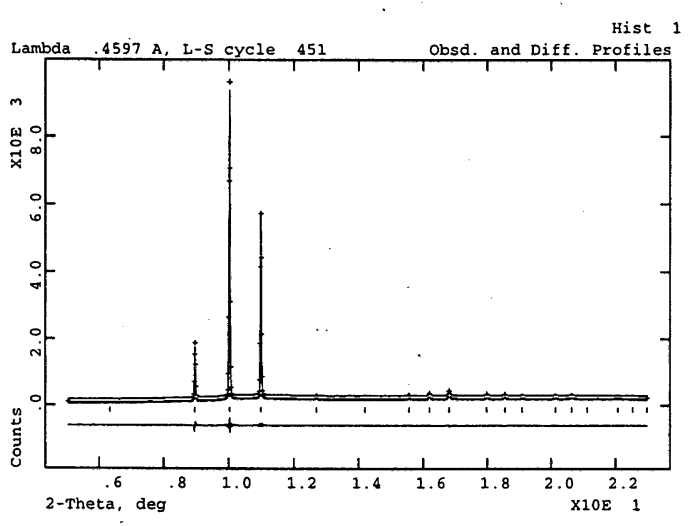

Fig. 1 Measured (crosses) and calculated (solid line) diffraction pattern of $\delta-\mathrm{N}_{2}$ at $9.5 \mathrm{GPa}$. The tickmarks show the positions of the reflections allowed by symmetry. The difference between the measured and the calculated patterns is shown below the tickmarks. $R_{p}$ and $R\left(F^{2}\right)$ of the fit are 0.02 and 0.03 , respectively. The refined lattice parameter is $\mathrm{a}=$ $5.8809(2) \AA$. The space group is $\mathrm{Pm} 3 \mathrm{n}$. Freely rotating nitrogen molecules were placed into the 2 (a) positions to simulate the spherically disordered molecules. Scattering factors were taken from [3]. The disklike disordered molecules were simulated by placing $1 / 4$ nitrogen atoms into the $16(\mathrm{i})$ positions.

The diffraction pattern of the new phase closely resembles the diffraction pattern of the $\delta$-phase resulting in earlier diffraction studies $[2,11]$ failing to detect the phase transition. Because of this strong resemblance we will call the new tetragonal phase $\delta^{*}$-phase. The phase transition is probably due to an orientational ordering of the disklike disordered molecules of the $\delta$-phase. Ordering of the disklike disordered molecules preceding the ordering of the spherically disordered molecules has been observed in a MD simulation by Nosé and Klein [5]. The relation between the lattice parameters of the $\delta^{*}$ and the $\delta$-phase suggests, that the spherically disordered molecules are situated at the corners and face centres of the tetragonal unit cell. A tetragonal space group allowing such an arrangement is $\mathrm{P}_{2} / \mathrm{ncm}$. The absence of a (031)-reflection ( $d=2.4609 \AA$ at $12.3 \mathrm{GPa}$ ) further supports the choice of $\mathrm{P}_{2} / \mathrm{ncm}$ as space group. Taking into account preferred orientation a structural model with freely rotating nitrogen molecules placed at the 4 (d) positions to simulate the spherically disordered molecules and rigid diatomic nitrogen bodies centred at the $4(\mathrm{e})(\mathrm{z} \approx 0.25)$ and $8(\mathrm{~g})(\mathrm{x} \approx 0.625)$ positions to simulate the ordered molecules produced good fits of the observed diffraction pattern (Fig. 2). Refined N-N intramolecular distances are $\mathrm{d}_{\mathrm{N} \cdot \mathrm{N}} \approx 1 \AA$ for the ordered molecules, a value reasonably close to the expected value $(1.09 \AA)$ considering the large thermal motion $\left(B \approx 5 \AA^{2}\right)$.

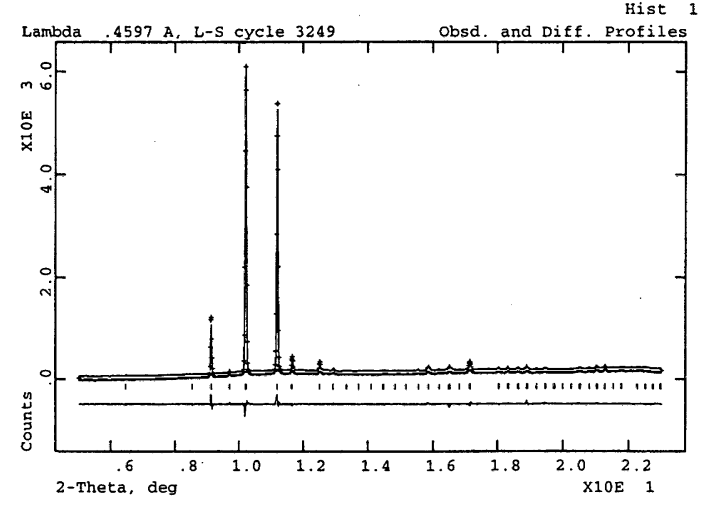

Fig. 2 Measured and calculated diffraction pattern of $\delta *-\mathrm{N}_{2}$ at $12.3 \mathrm{GPa} . \mathrm{R}_{\mathrm{p}}$ and $\mathrm{R}\left(\mathrm{F}^{2}\right)$ of the fit are 0.04 and 0.08 , respectively. The refined lattice parameters are $\mathrm{a}=8.1652(2)$ $\AA$ and $\mathrm{c}=5.7600$ (3) $\AA$. The space group is $\mathrm{P}_{2} / \mathrm{ncm}$. Freely rotating nitrogen molecules were placed into the $4(\mathrm{~d})$ positions to simulate the spherically disordered molecules. The ordered molecules were simulated by a rigid body with the nitrogen atoms placed into the $8(\mathrm{i})$ and $16(\mathrm{j})$ positions.

\begin{tabular}{llll}
\hline \multicolumn{2}{l}{ cubic $(9.5 \mathrm{GPa})$} & \multicolumn{2}{c}{ tetragonal $(12.3 \mathrm{GPa})$} \\
hkl & $\mathrm{d}(\AA)$ & hkl & $\mathrm{d}(\AA)$ \\
\hline 200 & 2.9405 & 220 & 2.8869 \\
& & 002 & 2.8800 \\
\hline- & - & 102 & 2.7160 \\
\hline 210 & 2.6300 & 310 & 2.5821 \\
& & 221 & 2.5809 \\
& & 112 & 2.5772 \\
\hline 211 & 2.4009 & 311 & 2.3562 \\
& & 202 & 2.3534 \\
\hline- & - & 212 & 2.2613 \\
\hline- & - & 321 & 2.1076 \\
\hline \hline
\end{tabular}

Tab. 1 Connection between the strongest reflections of $\delta-\mathrm{N}_{2}$ and $\delta^{*}-\mathrm{N}_{2}$.

At $16.5 \mathrm{GPa}$ another phase transition occurred. The observed diffraction pattern (Fig. 3) are in good agreement with the orientational ordered rhombohedral structure (R-3c) found by Mills et al. [2] at lower temperatures and pressures. No evidence for further structural phase transitions was observed up to $50 \mathrm{GPa}$.

\section{Conclusions}

We studied structural properties of molecular nitrogen between 5 and $50 \mathrm{GPa}$. Diffraction pattern with an extremely good sensitivity and resolution were measured due to the use of high intensity synchrotron radiation from a third generation source and of large image plates (A3) at large distances (up to $460 \mathrm{~mm}$ ). Structural parameters were refined. Values with an 
accuracy comparable to single crystal studies [3] were obtained.

The $\delta$-phase was found to be stable between 5 and $11 \mathrm{GPa}$ at room temperature. At $11 \mathrm{GPa}$ a phase transition to a tetragonal phase occured. Good agreement between measured and calculated diffraction pattern was achieved assuming, that the transition is due to an ordering of the disklike disordered molecules of the $\delta$-phase as suggested by MD calculations [5]. The space group of the new $\delta^{*}$-phase is presumably $\mathrm{P} 4_{2} / \mathrm{ncm}$. The phase transition is accompanied by an anomalous behavior of the vibrational spectrum of the $\delta$-phase [7].

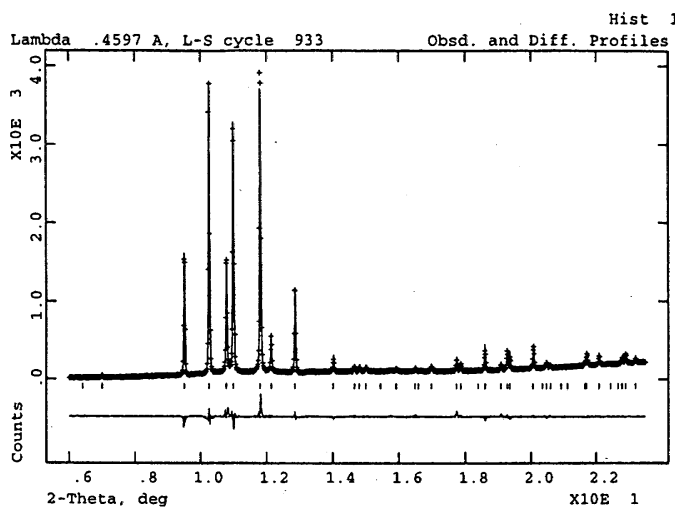

Fig. 3 Measured and calculated diffraction pattern of $\varepsilon-\mathrm{N}_{2}$ at 18.5 GPa. $R_{p}$ and $R\left(F^{2}\right)$ of the fit are 0.04 and 0.08 , respectively. The refined lattice parameters (hexagonal unit cell) are $\mathrm{a}=7.5269(2) \AA$ and $\mathrm{c}=10.5534(2) \AA$. The space group is R-3c. The (hexagonal) unit cell contains 24 molecules on two crystallographic positions. They were simulated by a rigid body with nitrogen atoms placed into the 12 (c) and $36(f)$ positions.

The phase transition to the $\varepsilon$-phase occurred at $16.5 \mathrm{GPa}$ Its structure is rhombohedral $(\mathrm{R}-3 \mathrm{c})$ as found by Mills et al. [2] at lower temperatures and pressures and not tetragonal as suggested by MC [4] and MD [5] calculations. The $\varepsilon$-phase exists to at least $50 \mathrm{GPa}$ at room temperature.

\section{References}

[1] A. F. Schuch and R. L. Mills, J. Chem. Phys., 52, 6000 (1970)

[2] R.L. Mills, B. Olinger, and D.T. Cromer, J. Chem. Phys., 84, 2837 (1986)

[3] D. T. Cromer, R. L. Mills, D. Schiferl, and L. A. Schwalbe, Acta Cryst. B, 37, 8 (1981)

[4] J. Belak, R. LeSar, and R.D. Etters, J. Chem. Phys., 92, 5430 (1990)

[5] S. Nosé and M. L. Klein, Phys. Rev. B, 33, 339 (1986)

[6] H. Schneider, W Häfner, A Wokaun, and H. Olijnyk, $J$. Chem. Phys., 96, 8046 (1992) and references therein

[7] M. I. M. Scheerboom and J. A. Schouten, Phys. Rev. Lett.,71, 2252 (1993)
[8] H. K. Mao, J. Xu, and P. M. Bell, J. Geophys. Res., 91, 4673 (1986)

[9] A. P. Hammersley, S. O. Svensson, M. Hanfland, A. N. Fitch, and D. Häusermann, High Pressure Research, 14, 235 (1996)

[10] A. C. Larson and R. B. von Dreele, GSAS - General structure analysis system, Los Alamos National Laboratory, LAUR $86-748$

[11] H. Olijnyk, J. Chem. Phys., 93, 8968 (1990) 